\title{
Verification of flow control protocols
}

Hansen, Klaus

Published in:

Computer Communication Review

Publication date:

1985

Document version

Publisher's PDF, also known as Version of record

Citation for published version (APA):

Hansen, K. (1985). Verification of flow control protocols. Computer Communication Review, 15(1). 
Verification of Elow control protocols.

\author{
Klaus Hansen
}

DIKU, University of Copenhagen

Sigurdsgade 41. DK-2200 Copenhagen N

\begin{abstract}
An important issue in computer network design is end-to-end control, a term covering error control and flow control. The validity of schemes for these purposes may be demonstrated using simple formal models, in which assertions about central program variables are proven. S. Krogdahl [2] and D.E.Knuth [1] have shown the validity of error control for a class of data link protocols using such methods. In this paper their method is extended to cover flow control. The method is illustrated by proving assertions for a simple case, and it is shown how complex systems may be seen as compositions of the simple case.
\end{abstract}

\title{
1. Introduction.
}

End-to-end protocols in computer networks guarantee that a data path between a producer (or data source) and a consumer (or data sink) provides a reliable service. An end-to-end protocol incorporates mechanisms for error control and flow control.

Error control covers the aspects of preserving the sequence as well as detecting loss, duplication or corruption of data. This is important when data are transfered over a data path with a significant probability for errors.

Flow control covers resource management. Transmission of data involves a certain amount of copying, and flow control methods are employed to utilize limited buffer resources. A flow control scheme is valid. when it ensures that transmission is always possible in spite of limited resources. uther aspects of flow control, i.e. smooth flow and good utilization of resources. are not treated in this paper. They involve statistical behaviour of the components, and a treatment calls for e.g. queueing theory, which is outside the scope of this paper.

The method used to verify schemes is similar to that in [1], A scheme is given by a set of algorithms working on a set of common variables. Each algorithms is given a name and is called an operation. The particular order in which the operations will be performed is immaterial for the purpose. To cite: 
"Our goal is to derive racts about any scheme that is based on these operations: it is in this sense we are studying a "protocol skeleton" for a large class of concelvable protocols. The facts we shall derive are expressed in terms of relations that remain invariant under all operations."

The scheme is thus completed by specifying a set of assertions about the variables.

The set of assertions consists of global and local assertions, the global assertions specifying the behaviour of combinations of pperations, and the local assertions determining the applicability of each operation.

The method is applied to a simple case and two composite cases. The cases are sumplified versions of protocols found in real networks: the $3 \mathrm{~m}-$ plification is done by ignoring the representation of various data. while keeping the essential parts of the protocols concerned with queves and feedback.

\section{Plow control.}

A constrained producer/consumer system (figure 1) consists of a producer of full buffers, a consumer that empties them, and two queves.

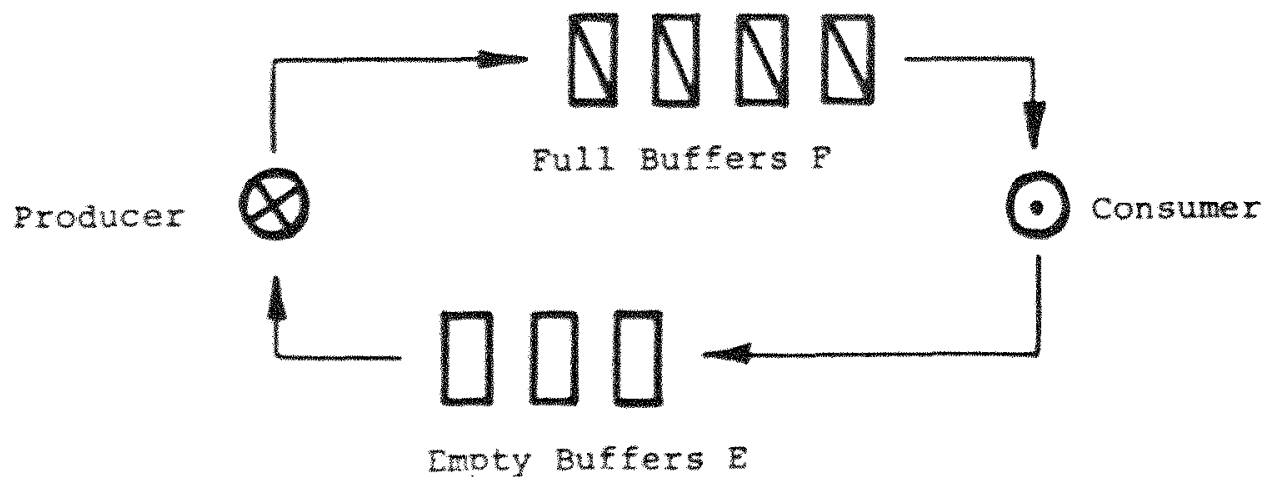

( $F$ denotes queue of full butfers, E queue of empty)

Figure 1: Constrained producer/consumer system.

Flow control is implicit in the synchronization method used, as a process is blocked (waits) whenever a queue is empty. The systen 19 stable irrespectively of the relative speeds; this is one of the purposes of synchronization methods.

However when the systen is split into two and transmisgion system is added (figure 2), it 13 necessary to provide a buffer pool at the consumer end, as the transmission path acts as a copying mechanism only, which cannot buffer data. We assume the transmission path to be error-iree and have $11 \mathrm{~m}-$ ited, but positive apeed. 


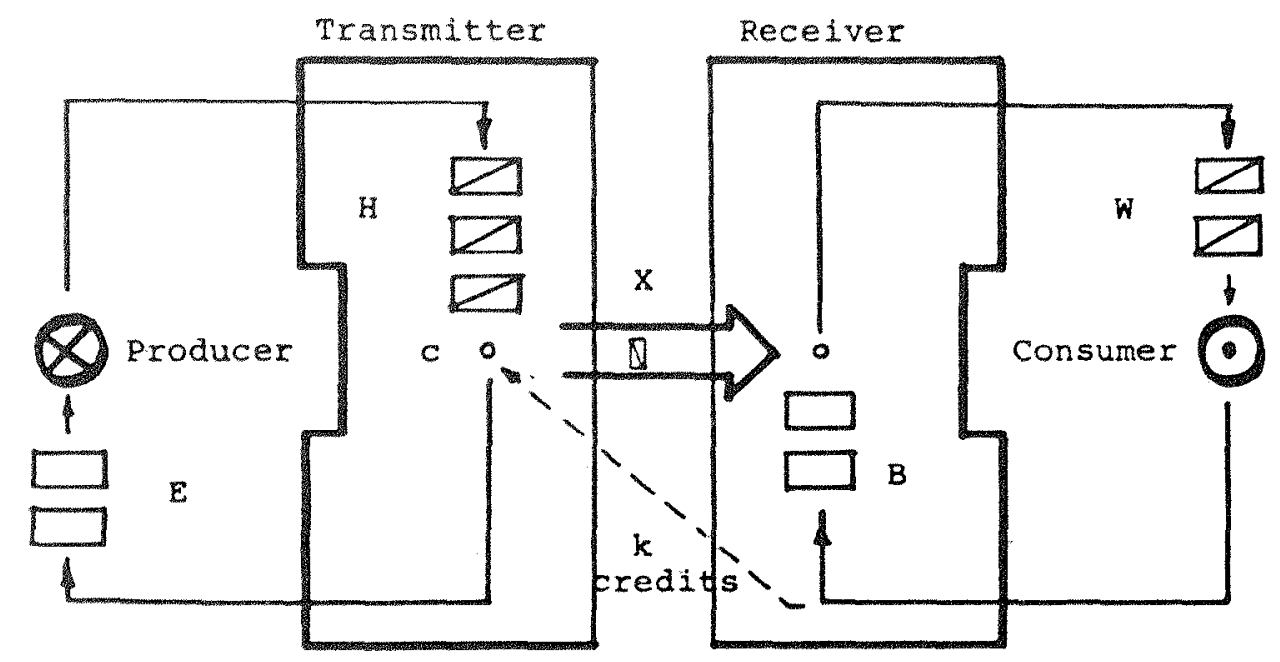

Note: lower case letters denote numbers. The queues are: $E$ empty buffers, $H$ buffers held beiore transmission, $B$ auxiliary pool of empty. W full buffers waiting to be consumed. $X$ is transmission path. $k$ credits in transit. $c$ available credits at $\mathrm{T}$.

Figure 2: Producer/consumer with transmission system.

The queue of full buffers ( $F$ ) has been split in three: buffers waiting for transmission $(H)$, buffers in transit $(X)$, and buffers delivered to the consumer, but waiting to be emptied ( $W$ ). The producer fills an empty buffer from $E$ and delivers it to the transmitter, where it is put into the $H$ queue. At an appropriate time, it is put into the transmission path, and the bufier is returned empty to $E$. When data arrives at the receiver, it is put into a buffer taken from $B$ and delivered to the consumer queue $W$. After emptyıng it. the consumer returns it to $B$. X may contain any number of buffers, depending on propagation time and speed of the data path.

The feed-back needed is provided by a stream of credits that conveys information about empty buffers, one credit representing one empty buffer.

The purpose of flow control is now to avoid the situation where $B$ is empty when data are received, an unrecoverable situation. If the number of buffers in $X$ and $B$ are called $X$ and $b$ respectively, this may be expressed as

$$
x \leq b
$$

1.e. the number of buffers in transit must not exceed the current size of $B$. The full description of the variables is: 


\begin{tabular}{|c|c|c|}
\hline & size of empty queve (E) & indialdy \\
\hline$h$ & size of hold queve $(H)$ & inteally \\
\hline$x_{x}$ & number of butfers in transit & Intaliy \\
\hline 0 & slze of (ree queve (B) & notialy \\
\hline$y$ & S3ze of queve or watho (w) & andediy \\
\hline & credits in transit & netally \\
\hline & curcent credits at transmitter & netal \\
\hline
\end{tabular}

\section{Operations and assertions for the simple system.}

Using the varlables described above, we can que a scheme ror the simple system in figure 2 that has tive operations, three for the transmitter (TI. T2, T3) and two for the recelver (R1. R2). The operations are quven below. The comments enclosed in ( and shows the semantics of the statement. which is otherwise only specified by the effect on the common variables. Local assertions are shown for each operation, and have to be true for trie operation to be applicable. e.g. $h>0$ and $c>0$ for a butier to be transmitted by operation T2. A protocol using Elow control wil consist of some sequence of these operations: a sequence is valid only if the local assertions are true when an operation is invoked.

\section{T1: PUT FULL. \\ (* assert e>0 *) \\ $e:=e-1 ;$ \\ $h:=h+1 ;$}

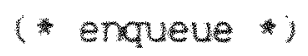

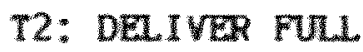

(*asert $h>0$ )

$$
\begin{array}{ll}
h:=h-1 ; & \text { (* dequeue }) \\
c:=c-1: & \text { (* SEND DATA } \\
x:=x+1 ; & \text { (* deliver empty*) }
\end{array}
$$

\section{T3: CREDIT ARRIVED}

(*asert $k>0$ *)

$k:=k-1:$

$c:=c+1 *$

\section{R1: FULL ARRIVED}

( assert $x>0$ )

$$
x:=x+1 ;
$$

$b:=b-1:$ (dequeve *)

$w_{*}^{*}=w_{1}$; ( deliver full

R2: PUT ENTT

(*asert a>0)

$y:=4-1$ :

$b ;=b+1 *$

$k:=k+1$; 
The global assertions are as follows. All variables except $c$ are nonnegative: For $c$ a weaker assumption $c+k \geq 0$ may be used (the meaning of this is not elaborated here). First, the purpose of Elow control is reformulated as

$$
c+k=b-x
$$

Second, no buffers may be lost or misplaced at $T$ or $R$ :

$$
\begin{aligned}
& e+h=\tilde{e} \\
& b+b=5
\end{aligned}
$$

It is easily seen that the assertions are true for the initial values and invariant under the operations. To prove $x \leq b$ observe that

$$
\mathrm{b}-\mathrm{x}=\mathrm{c}+\mathrm{k} \text { and } \mathrm{c}+\mathrm{k} \geq 0
$$

which implies that

$$
x-b \leq 0
$$

The global assertions will then be true, and the total scheme be valid.

A simplified version of the operations is worth considering. When the variables $c$ and $k$, and operation $T 3$ are omitted. there 13 no feed-back. If the consumer and transmission path are faster than the consumer, it is easy to construct a valid sequence which violates equation (1.1): an example 13 (T1 T2 R1) repeated a sufficient number of times. This scheme is invalid for flow control; but it may of course be used if one dares to use timing considerations in validity arguments.

\section{Composite systems.}

Two common ways if obtaining more complex schemes are to combine simple schemes by concatenation or nesting, in the following called serial and multiplexed flow control.

Serial flow control is found in gateways between different nets and in front-ends for host computers.

Multiplexing and the resulting nesting of flow control is common, examples are the X.25 datalink and DCE-DTE levels. or the datalink, network and transport layers in the Open systems Interconnection model [3].

\section{Serial flow control.}

Figure 3 shows the concatenation of two schemes. The arguments above are easily generalized to a concatenation of simple schemes joined in the obvious way. The five operations are still valid, with a change of variables to indexed variables, to distinguish between the component schemes. As a consequence, some variable names are synonyms: 


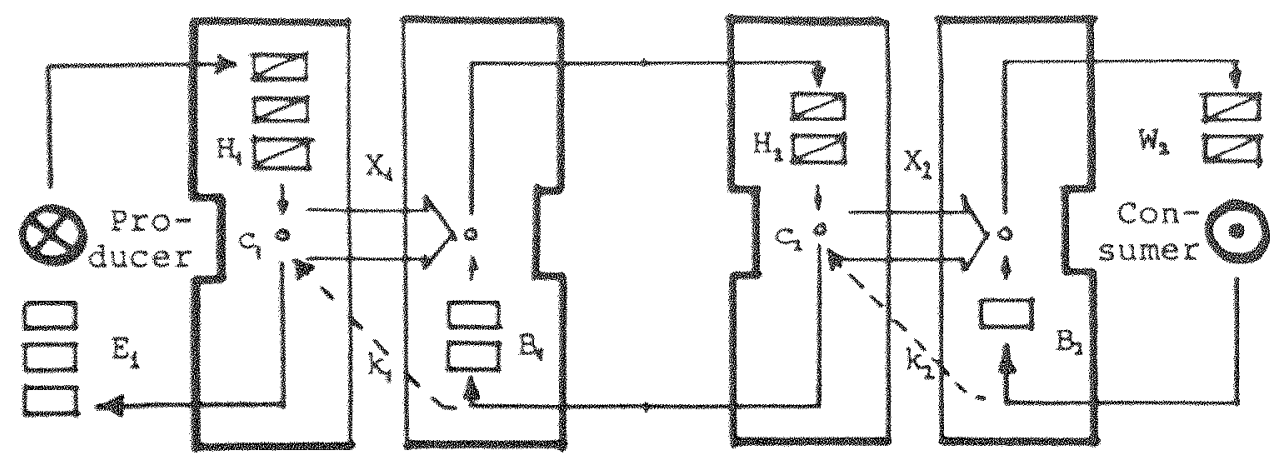

Figure 3: Serial flow control.

$$
b_{1}=e_{2} \text { and } w_{1}=h_{2}
$$

The operations are derived from those of the simple system by indexing of variables. The assertions are ( 1 having values 1 and 2 ):

$$
\begin{gathered}
c_{1}+k_{1}=b_{1}-x_{1} \\
e_{1}+h_{1}=\tilde{e}_{1} \\
w_{1}+b_{1}=b_{1}
\end{gathered}
$$

It is easily verified that the assertions are valid. The proot of $x_{\text {in }} \leq \mathrm{b}$ is similar to that of the simple scheme. The conclusion is that concatenation of Elow control is a meaningful operation, which preserve the validity.

\section{Multiplexing.}

When several data streans share a channel, but are otherwise independent, the data streans are said to be multiplexed (see also [3]). The outer transmission systems (for the streans) use that of the inner (the channel) for the actual transfer (see Elgure 4). As both the channel and the streams need flow control, this leads to nested flow control. The combined producers and stream transmitters act as one producer for the channel, and similarily the combined consumers and receivers act as one consumer.

In the following the index zero signifies the channel varlables. and a positive index the corresponding stream. The notation is otherwise unchanged.

The scheme has been simplified somewhat with respect to the queues. It is assumed that no copying is needed at the stream/channel interface. As a consequence $E_{0}$ and $B$, are omitted. Similarily the buffers in $B$ are nonexistent, but $b_{1}$ is the number of buffers in $B_{0}$ that strean 1 considers its 


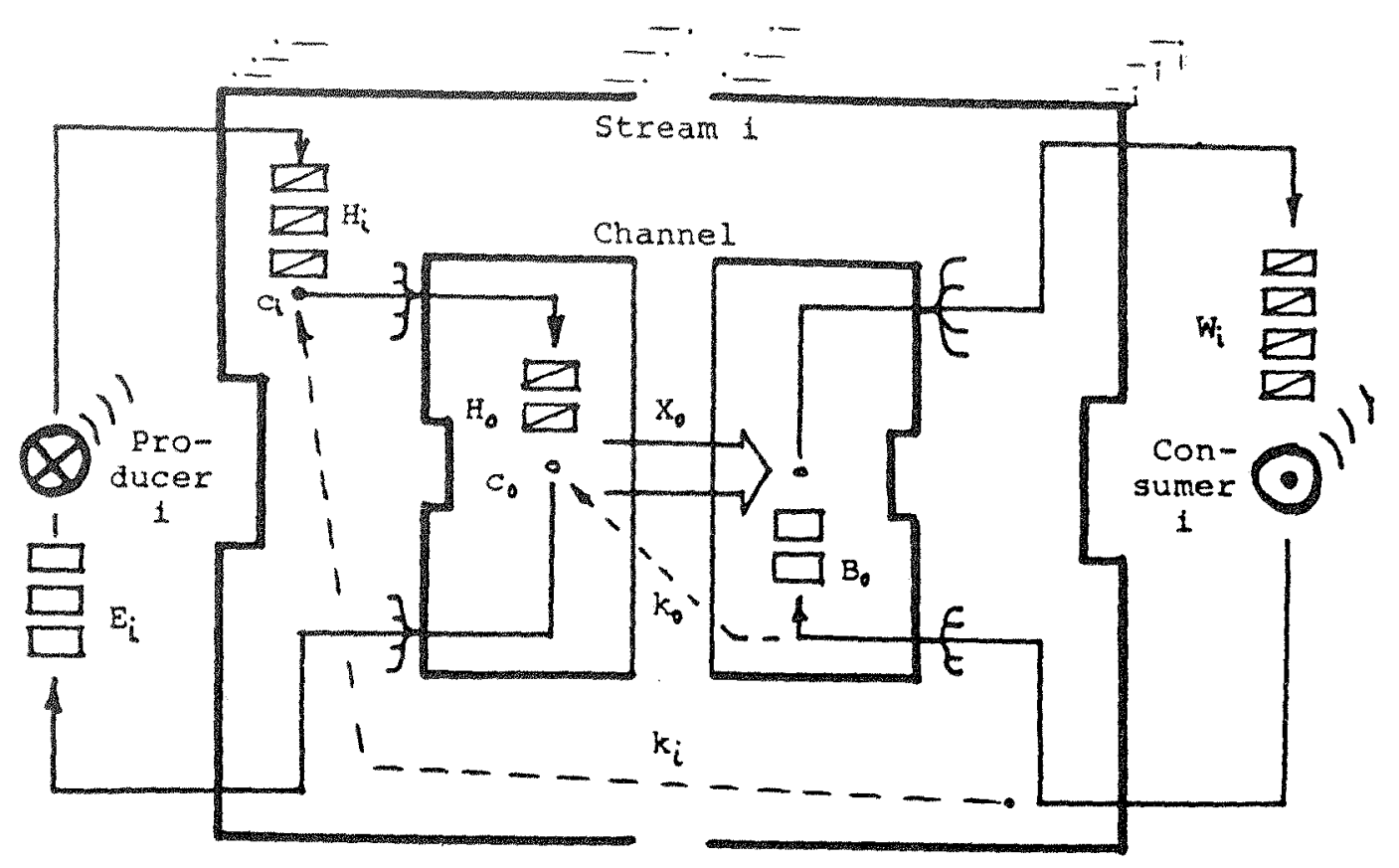

Figure 4: Multiplexing, nested ilow control.

share. Allocation of buffers is a separate 1ssue.

The operations of the channel and the streams are as follows inote that some actions are coupled together):

Operations of the channel.

T1: PUT FULL.

(* assert nil *)

$$
h_{0}:=h_{0}+1 ; \quad \text { (* enqueue } * \text { ) }
$$

T2: DELIVER FULL.

(* assert $h_{0}>0$ *)

$\mathrm{h}_{0}:=\mathrm{h}_{0}-1$

(* dequeue *)

$c_{0}:=0-1$ :

(* SEND DATA *)

call SIREAM .T4; (* deliver empty *)

T3: CREDIT ARRIVED

(* assert $k_{0}>0$ *)

$k_{0}:=k_{0}-1$
$c_{0}:=c_{0}+1$

R1: FULL ARRIVED

(* assert $x_{0}>0 *$ )

$\mathrm{x}_{0}:=\mathrm{x}_{0}-1:$
$\mathrm{b}_{0}:=\mathrm{b}_{0}-1 ;$$\quad$ (* dequeue *)

(* call $\operatorname{STREAM}_{2} \cdot \mathrm{Rl} *$ *)

\section{R2: PUT EAPTY}

(* assert nil *)

$$
\begin{array}{ll}
b_{0}:=b_{0}+1 ; & (* \text { enqueue } *) \\
k_{0}:=k_{0}+1 ; & \text { (* SEND CREDIT *) }
\end{array}
$$


operations for streas 1 .

1. PUt FUL

( assert $e_{1}>0$ )

$$
\begin{aligned}
& e_{1}:=e_{1}{ }^{-1}: \\
& h_{1}:=h_{1}+1:
\end{aligned}
$$

T2: DEL IVER FULL

( assert $h,>0 *$ )

$$
\begin{aligned}
& n_{1}:=n_{1} \geq_{1}: \quad \text { ( dequeve *) } \\
& c_{1}^{2}:=c^{n}-1: \\
& x_{1}:=x_{1}^{2}+1: \quad \text { ( call CHANNEL. T1 }
\end{aligned}
$$

T3: CREDIT ARRIVED

(* assert $k_{i}>0$ )

$$
\begin{aligned}
& k_{1}:=k_{1}^{1}-1: \\
& c_{1}:=c_{1}+1:
\end{aligned}
$$

T: ERPTY ARRIVED

( assert $n i l$ )

$$
e_{1}:=e_{1}+1 ; \quad \text { (* deliver *) }
$$

R1: FULL ARRIVED

( assert $x>0$ )

$$
\begin{aligned}
& x_{1}:=x_{1}-1: \\
& b_{1}^{1}:=b_{i}^{-1}: \\
& w_{1}:=w_{1}^{1}+1: \quad \text { (* deliver full }
\end{aligned}
$$

R2: PUT ERPTY

(* assert ${ }_{1}>0$ )

H: $=\omega_{1}=1$;

$E^{1}:=\epsilon_{i}^{1}+1 ;$

CE11 CHANNEL.R2:

$k_{i}:=k_{i}+1$;

(* SEND CREDIT *)

The invariants are (note $1>0$ unless otherwise stated):

$$
\begin{gathered}
c_{1}+k_{1}=b_{1}-x_{1} \quad(a 11 i) \\
\Sigma\left(e_{1}+h_{1}\right)+h_{0}=\Sigma \tilde{e}_{1} \\
\Sigma w_{1}+b_{0}=\delta_{0}
\end{gathered}
$$

Muliplexing is consistent

$$
\Sigma x_{1}=h_{0}+x_{0}
$$

It is easily verified that the assertions are true under the operations. If $\Sigma b_{1} \leq b_{0}$ then follows (as $\Sigma b_{1} \leq b_{0}$ is invariant): 


$$
\begin{gathered}
b_{1}-x_{1}=c_{i}+k_{2} \geq 0 \\
\text { which implies } b_{i} \geq x_{1} \\
\text { Which implies } b_{0} \geq \Sigma b_{i} \geq \Sigma x_{1}=h_{0}+x_{0} \geq x_{0}
\end{gathered}
$$

As this does not involve the assertions for $c_{0}$ and $k_{0}$, it shows that independent of the channel Elow control mechanism, the channel will never overflow if the stream Elow control is conservative enough. Thus interesting enough, we have shown that the channel flow control can be omitted. This may be taken as an argument against indiscriminate use of nested or layered systems.

\section{Conclusion.}

It has been shown that a simple scheme consisting of a iew timeindependent operations and their associated variables and assertions may be used to validate the essential part of flow control protocols. Furthermore, it is possible to obtain results for composite systems using the same method.

The method combines well with the error control validation of [1], to give a total method for verifying end-to-end protocols.

other methods for specification and verification [4] like finite-state automata or Petri nets are used for specific protocols to prove e.g. ireedom from deadlock, completeness, or stability, or to verify that the design meets its specification. The approach in this paper is not quite the same, as it is more limted with respect to properties, and more general in scope, as the properties are shown for classes of conceivable protocols.

\section{Acknouledgements.}

I wish to thank Jens Hammerum for the original idea, which appeared in his Master's thesis in disguise, and A.P. Ravn for much advice on the presentation of the ideas.

\section{Litterature.}

[1] D.E.Knuth: Verification of link-level protocols. BIT 21,1 (1981)

[2] S.Krogdahl: Verification of a class of link-level protocols. BIT 18,4 (1978)

[3] ISO/IS 7498

Open Systems Interconnection

(1983)

[4] C.A.Sunishine: Formal Modelling of Communication Protocols. USC/Information Sciences Institute, RR-81-89 (March 1981) 